\title{
ON THE DETERMINATION OF SURFACES CAPABLE OF CONFORMAL REPRESENTATION UPON THE PLANE IN SUCH A MANNER THAT GEODETIC LINES ARE REPRESENTED BY
} ALGEBRAIC CURVES*

BY

\author{
HENRY FREEMAN STECKER
}

\section{Introduction.}

BeLtram has shown $\uparrow$ that surfaces of constant curvature can be built upon the plane in such a manner that the geodetic lines shall go over into straight lines, and that this result is true for no other surface. He considered this as the simplest case of building one surface upon another in sueh a manner that the geodetic lines of one surface shall go over into the geodetic lines of the other. The general question was later solved by DiNI. $\ddagger$ It is an immediate consequence of BeLtRami's memoirs that surfaces of constant curvature are the only surfaces that can be built conformally upon the plane in such a manner that the geodetic lines shall go over into straight lines or ares of circles. $\S$ This latter fact suggests a generalization of BELTRAMI's problem different from the one which he had in mind, i. e., to so build a surface conformally upon the plane in such a manner that the geodetic lines shall go over into algebraic curves. It is proposed to consider that question.

\section{$\S 1$.}

We consider a doubly infinite system of algebraic curves in the plane :

$$
F_{3}(x, y)+A F_{2}(x, y)+B F_{1}(x, y)=0,
$$

where $A$ and $B$ are the parameters of the system.

We think of the surface as given by

$$
x_{i}=\phi_{i}(\mu, \nu) \quad(i=1,2,3),
$$

* Presented to the Society February 23, 1901. Received for publication November 20, 1900.

† Annali di Matematica, vol. 7, 1866.

$\ddagger$ Annali di Matematica, ser. 2, vol. 3, 1870.

$\S$ For an independent proof of this proposition see F. Busse, Inaugural Dissertation, Berlin, 1896. 
where $\mu$ and $\nu$ are rectangular surface coördinates, i. e., such that the systems of curves $\mu=$ const., $\nu=$ const., intersect orthogonally.

Let the relation between the surface and the plane be given by

$$
x=\psi_{1}(\mu, \nu), \quad y=\psi_{2}(\mu, \nu) .
$$

Then we write $f_{i}(\mu, \nu)$ for $F_{i}\left(\psi_{1}, \psi_{2}\right)$ and equation (1) becomes

$$
f_{3}(\mu, \nu)+A f_{2}(\mu, \nu)+B f_{1}(\mu, \nu)=0,
$$

and from this we find:

$$
\begin{aligned}
& f_{3}^{\prime}(\mu, \nu)+A f_{2}^{\prime}(\mu, \nu)+B f_{1}^{\prime}(\mu, \nu)=0, \\
& f_{3}^{\prime \prime}(\mu, \nu)+A f_{2}^{\prime \prime}(\mu, \nu)+B f_{1}^{\prime \prime}(\mu, \nu)=0,
\end{aligned}
$$

where

$$
\begin{gathered}
f_{i}^{\prime}(\mu, \nu)=\frac{\partial f_{i}(\mu, \nu)}{\partial \mu} d \mu+\frac{\partial f_{i}(\mu, \nu)}{\partial \nu} d \nu \\
f_{i}^{\prime \prime}(\mu, \nu)=\frac{\partial^{2} f_{i}(\mu, \nu)}{\partial \mu^{2}} d \mu^{2}+2 \frac{\partial^{2} f_{i}(\mu, \nu)}{\partial \mu \partial \nu} d \mu d \nu+\frac{\partial^{2} f_{i}(\mu, \nu)}{\partial \nu^{2}} d \nu^{2} \\
+\frac{\partial f_{i}(\mu, \nu)}{\partial \mu} d^{2} \mu+\frac{\partial f_{i}(\mu, \nu)}{\partial \nu} d^{2} \nu
\end{gathered}
$$

From (2), (3) and (4) we obtain

We write

$$
\left|\begin{array}{lll}
f_{1} & f_{2} & f_{3} \\
f_{1}^{\prime} & f_{2}^{\prime} & f_{3}^{\prime} \\
f_{1}^{\prime \prime} & f_{2}^{\prime \prime} & f_{3}^{\prime \prime}
\end{array}\right|=0 .
$$

$$
n_{i 1}=\frac{\partial f_{i}}{\partial \mu}, \quad n_{i 2}=\frac{\partial f_{i}}{\partial \nu} ; \quad m_{i 1}=\frac{\partial^{2} f_{i}}{\partial \mu^{2}}, \quad m_{i 2}=\frac{\partial^{2} f_{i}}{\partial \nu^{2}}, \quad m_{i 3}=\frac{\partial^{2} f_{i}}{\partial \mu \partial \nu} .
$$

Making use of these abbreviations, substituting for $f_{i}^{\prime}$ and $f_{i}^{\prime \prime}$ their values, and expanding in terms of the first row, we have from equation (5):

or

$$
\begin{array}{r}
f_{1}\left|\begin{array}{ll}
n_{21} d \mu+n_{22} d \nu & m_{21} d \mu^{2}+m_{22} d \nu^{2}+2 m_{23} d \mu d \nu+n_{21} d^{2} \mu+n_{22} d^{2} \nu \\
n_{31} d \mu+n_{32} d \nu & m_{31} d \mu^{2}+m_{32} d \nu^{2}+2 m_{33} d \mu d \nu+n_{31} d^{2} \mu+n_{32} d^{2} \nu
\end{array}\right| \\
-f_{2}\left|\begin{array}{ll}
n_{11} d \mu+n_{12} d \nu & m_{11} d \mu^{2}+m_{12} d \nu^{2}+2 m_{13} d \mu d \nu+n_{11} d^{2} \mu+n_{12} d^{2} \nu \\
n_{31} d \mu+n_{32} d \nu & m_{31} d \mu^{2}+m_{32} d \nu^{2}+2 m_{33} d \mu d \nu+n_{31} d^{2} \mu+n_{32} d^{2} \nu
\end{array}\right| \\
+f_{3}\left|\begin{array}{ll}
n_{11} d \mu+n_{12} d \nu & m_{11} d \mu^{2}+m_{12} d \nu^{2}+2 m_{13} d \mu d \nu+n_{11} d^{2} \mu+n_{12} d^{2} \nu \\
n_{21} d \mu+n_{22} d \nu & m_{21} d \mu^{2}+m_{22} d \nu^{2}+2 m_{23} d \mu d \nu+n_{21} d^{2} \mu+n_{22} d^{2} \nu
\end{array}\right|=0,
\end{array}
$$

$$
f_{1} \theta(23)-f_{2} \theta(13)+f_{3} \theta(12)=0,
$$


where

$$
\begin{aligned}
\theta(23) & =\left|\begin{array}{c}
n_{21} m_{21} \\
n_{31} m_{31}
\end{array}\right| d \mu^{3}+\left|\begin{array}{c}
n_{21} m_{22} \\
n_{31} m_{32}
\end{array}\right| d \mu d \nu^{2}+2\left|\begin{array}{c}
n_{21} m_{23} \\
n_{31} m_{33}
\end{array}\right| d \mu^{2} d \nu+\left|\begin{array}{c}
n_{21} n_{22} \\
n_{31} n_{32}
\end{array}\right| d \mu d^{2} \nu \\
& +\left|\begin{array}{c}
n_{22} m_{21} \\
n_{32} m_{31}
\end{array}\right| d \mu^{2} d \nu+\left|\begin{array}{c}
n_{22} m_{22} \\
n_{32} m_{32}
\end{array}\right| d \nu^{3}+2\left|\begin{array}{c}
n_{22} m_{23} \\
n_{32} m_{33}
\end{array}\right| d \mu d \nu^{2}+\left|\begin{array}{c}
n_{22} n_{21} \\
n_{32} n_{31}
\end{array}\right| d \nu d^{2} \mu,
\end{aligned}
$$

with corresponding expressions for $\theta(13), \theta(12)$. Adding like terms, we have for terms in $d \mu^{3}$ :

or

$$
\left\{f_{1}(\mu, \nu)\left|\begin{array}{l}
n_{21} m_{21} \\
n_{31} m_{31}
\end{array}\right|-f_{2}(\mu, \nu)\left|\begin{array}{l}
n_{11} m_{11} \\
n_{31} m_{31}
\end{array}\right|+f_{2}(\mu, \nu)\left|\begin{array}{l}
n_{11} m_{11} \\
n_{21} m_{21}
\end{array}\right|\right\} d \mu^{3},
$$

$$
\left|\begin{array}{lll}
f_{1}(\mu, \nu) & n_{11} & m_{11} \\
f_{2}(\mu, \nu) & n_{21} & m_{21} \\
f_{3}(\mu, \nu) & n_{31} & m_{31}
\end{array}\right| d \mu^{3} .
$$

And we easily see that we have like determinants for the coefficients of the other terms. Indeed if we write [11] for the coefficient of $d \mu^{3}$, our differential equation is :

where $\Delta$ is the determinant $\left|f_{1} n_{21} n_{32}\right| \cdot$

$$
[11] d \mu^{3}+[22] d \nu^{3}+\{2[13]+[21]\} d \mu^{2} d \nu+\{2[23]+[12]\} d \mu d \nu^{2}
$$

$$
+\Delta\left(d \mu d^{2} \nu-d \nu d^{2} \mu\right)=0,
$$

For shortness we write equation (7) in the form :

$$
a_{1} d \mu^{3}+a_{4} d \nu^{3}+a_{2} d \mu^{2} d \nu+a_{3} d \mu d \nu^{2}+a_{5}\left(d \mu d^{2} \nu-d \nu d^{2} \mu\right)=0 \text {. }
$$

$\S 2$.

The geodetic lines of our surface are given by the differential equation:

$$
\begin{aligned}
\frac{1}{2} G \frac{\partial G}{\partial \mu} d \nu^{3} & -\frac{1}{2} E \frac{\partial E}{\partial \nu} d \mu^{3}+\left[E \frac{\partial G}{\partial \mu}-\frac{1}{2} G \frac{\partial E}{\partial \mu}\right] d \mu^{2} d \nu \\
+ & {\left[G \frac{\partial E}{\partial \nu}-\frac{1}{2} E \frac{\partial G}{\partial \nu}\right] d \mu d \nu^{2}+E G\left(d \mu d^{2} \nu-d \nu d^{2} \mu\right)=0 ; }
\end{aligned}
$$

we are to compare this with equation (8). This comparison gives the following system of partial differential equations :

$$
\begin{gathered}
E \frac{\partial G}{\partial \mu}-\frac{1}{2} G \frac{\partial E}{\partial \mu}=\lambda a_{2}, \quad G \frac{\partial E}{\partial \nu}-\frac{1}{2} E \frac{\partial G}{\partial \nu}=-\lambda a_{3}, \\
\frac{1}{2} G \frac{\partial G}{\partial \mu}=\lambda a_{4}, \quad \frac{1}{2} E \frac{\partial E}{\partial \nu}=-\lambda a_{1}, \quad E G=\lambda a_{5} .
\end{gathered}
$$


From this system we eliminate $\lambda$ by means of the last equation and obtain:

$$
\begin{gathered}
E \frac{\partial G}{\partial \mu}-\frac{1}{2} G \frac{\partial E}{\partial \mu}=E G \frac{a_{2}}{a_{5}}, \quad G \frac{\partial E}{\partial \nu}-\frac{1}{2} E \frac{\partial G}{\partial \nu}=-E G \frac{a_{3}}{a_{5}}, \\
\frac{1}{2} G \frac{\partial G}{\partial \mu}=E G \frac{a_{4}}{a_{5}}, \quad \frac{1}{2} E \frac{\partial E}{\partial \nu}=-E G \frac{a_{1}}{a_{5}}
\end{gathered}
$$

From these we have :

1.

$$
\begin{aligned}
& \frac{\partial \log G}{\partial \mu}-\frac{1}{2} \frac{\partial \log E}{\partial \mu}=\frac{a_{2}}{a_{5}}, \\
& \frac{\partial \log E}{\partial \nu}-\frac{1}{2} \frac{\partial \log G}{\partial \nu}=-\frac{a_{3}}{a_{5}},
\end{aligned}
$$

2.

3.

$$
\begin{aligned}
& \frac{\partial \log E}{\partial \nu}=-2 \frac{a_{1}}{a_{5}} \frac{G}{E}, \\
& \frac{\partial \log G}{\partial \mu}=2 \frac{a_{4}}{a_{5}} \frac{E}{G} .
\end{aligned}
$$

Integrating 1 and 2 we have :

1.

$$
\log G-\frac{1}{2} \log E=\log F_{1}+\log V_{1},
$$

2.

$$
\log E-\frac{1}{2} \log G=-\log F_{2}+\log U_{1},
$$

where $F_{1}=e^{\int \frac{a_{2}}{a_{5}} d \mu}$ and $F_{2}=e^{\int \frac{a_{3}}{a_{5}} d \mu} ; U_{1}$ is a function of $\mu$ only and $V_{1}$ of $\nu$ only. Then (13) gives

1.

$$
\frac{G}{E^{\frac{1}{2}}}=F_{1}^{\prime} V_{1}
$$

2.

$$
\frac{E}{G^{\frac{1}{2}}}=F_{2}^{-1} U_{1}
$$

Squaring each of these equations and multiplying it by the other we find

1.

$$
\begin{aligned}
& E=U_{1}^{4} V_{1}^{3} F_{1}^{3} F_{2}^{-\frac{4}{3}}, \\
& G=U_{1}^{3} V_{1}^{4} F_{1}^{4} F_{2}^{-\frac{3}{3}} .
\end{aligned}
$$

2.

From these last two equations we have the following values:

1.

$$
\frac{E}{G}=U_{1}^{\frac{2}{3}} V_{1}^{-\frac{2}{3}} F_{1}^{-\frac{2}{3}} F^{-}
$$

(16) 2.

2. $\quad \frac{\partial \log G}{\partial \mu}=\frac{2}{3} \frac{1}{U_{1}} \frac{d U_{1}}{d \mu}+\frac{4}{3} \frac{a_{2}}{a_{5}}-\frac{2}{3} \frac{\partial \log F_{2}}{\partial \mu}$,

3. $\quad \frac{\partial \log E}{\partial \nu}=\frac{2}{3} \frac{1}{V_{1}} \frac{d V_{1}}{\partial \nu}-\frac{4}{3} \frac{a_{3}}{a_{3}}+\frac{2}{3} \frac{\partial \log F_{1}}{\partial \nu}$. 
We substitute these values in equations (3) and (4) of the system (12) and obtain :

$$
\begin{aligned}
& \frac{d U_{1}}{d \mu}+\left[2 \frac{a_{2}}{a_{5}}-\frac{\partial \log F_{2}^{\prime}}{\partial \mu}\right] U_{1}=3 \frac{a_{4}}{a_{5}} V_{1}^{-\frac{3}{3}} F_{1}^{-\frac{3}{3}} F_{2}^{-3} U_{1}^{\xi}, \\
& \frac{d V_{1}}{d \nu}+\left[-2 \cdot \frac{a_{3}}{a_{5}}+\frac{\partial \log F_{1}}{\partial \nu}\right] V_{1}=-3 \frac{a_{1}}{a_{5}} U_{1}^{-3} F_{1}^{3} F_{2}^{\xi_{3}^{3}} V_{1}^{\xi}
\end{aligned}
$$

The integrals of these are:

1.

2.

$$
\begin{aligned}
& \frac{V_{1}^{3}}{U_{1}^{\frac{3}{3}}}=2 F_{1}^{\frac{3}{3}} F_{2}^{-\frac{3}{3}}\left(F_{3}+V_{2}\right), \\
& \frac{U_{1}^{3}}{V_{1}^{3}}=2 F_{1}^{3} F_{2}^{-\frac{3}{3}}\left(F_{4}+U_{2}\right),
\end{aligned}
$$

where

$$
F_{3}=\int-\frac{a_{4}}{a_{5}} F_{1}^{-2} d \mu, \quad F_{4}=\int \frac{a_{1}}{a_{5}} F_{2}^{2} d \nu
$$

and $U_{2}$ and $V_{2}$ are functions of $\mu$ only and of $\nu$.only respectiyely.

These equations are sufficient to say whether or not a solution exists and to find such solutions if they do exist. It is proposed to illustrate this by an example (pp. 156-159) before going farther with the general theory.

EXAMPLE. We take $f_{3}=f_{3}(\nu)$, a function of $\nu$ only; also

$$
f_{2}(\mu, \nu)=\mu^{n} \nu^{m}, f(\mu, \nu)=\mu^{n} \nu^{\beta} .
$$

Then after some calculation we find the following values:

$$
\begin{aligned}
& a_{1}=0, \\
& a_{2}=\mu^{2 n-2} \nu^{\beta+m-1} n(n+1)(\beta-m) f_{3}(\nu), \\
& a_{3}=\mu^{2 n-1} \nu^{\beta+m-2}\left[n(\beta-m)(m+\beta-1) f_{3}(\nu)-2 n(\beta-m) \nu \frac{d f_{3}(\nu)}{d \nu}\right], \\
& a_{4}=\mu^{2 n} \nu^{\beta+m-3}(\beta-m)\left[\beta m f_{3}(\nu)-(\beta+m-1) \nu \frac{d f_{3}(\nu)}{d \nu}+\nu^{2} \frac{d^{2} f_{3}(\nu)}{d \nu^{2}}\right], \\
& a_{5}=\mu^{2 n-1} \nu^{\beta+m-1} n(\beta-m) f_{3}(\nu) .
\end{aligned}
$$

From these we find:

$$
\begin{aligned}
& \frac{a_{2}}{a_{5}}=\frac{n+1}{\mu}, \\
& \frac{a_{3}}{a_{5}}=\frac{m+\beta-1}{\nu}-2 \frac{1}{f_{3}(\nu)} \frac{d f_{3}(\nu)}{d \nu}, \\
& \frac{a_{1}}{a_{5}}=0, \\
& \frac{a_{4}}{a_{5}}=\frac{\mu}{n}\left[\frac{\beta m}{\nu^{2}}-(\beta+m-1) \frac{1}{\nu} \frac{1}{f_{3}(\nu)} \frac{d f_{3}(\nu)}{d \nu}+\frac{1}{f_{3}(\nu)} \frac{d^{2} f_{3}(\nu)}{d \nu^{2}}\right]=\frac{\mu}{n} \theta(\nu) .
\end{aligned}
$$


Then we have:

$$
\begin{aligned}
& \int \frac{a_{2}}{a_{5}} d \mu=\int(n+1) \frac{d \mu}{\mu}=\log \mu^{n+1} \\
& \int \frac{a_{3}}{a_{5}} d \nu=\int(m+\beta-1) \frac{d \nu}{\nu}-2 \int d \log f_{3}(\nu) d \nu=\log \frac{\nu^{m+\beta-1}}{f_{3}^{2}(\nu)} .
\end{aligned}
$$

Therefore

$$
\begin{gathered}
F_{1}=\mu^{n+1}, \quad F_{2}=\frac{\nu^{m+\beta-1}}{f_{3}^{2}(\nu)}, \quad F_{4}=0 \\
F_{3}=\int-\frac{a_{4}}{a_{5}} F_{1}^{-2} d \mu=\int-\theta(\nu) \mu^{-2(n+1)+1} d \mu=\frac{\theta(\nu)}{2 n^{2}} \mu^{-2 n}
\end{gathered}
$$

Introducing these values into (19) we have:

1. $V_{1}^{3} U_{1}^{-\frac{3}{3}}=2 \mu^{\frac{4}{3}(n+1)} \nu^{-\frac{2}{3}(m+\beta-1)} f_{3}^{\frac{3}{3}}(\nu)\left[\frac{\theta(\nu) \mu^{-2 n}+2 n^{2} V_{2}}{2 n^{2}}\right]$

2. $\quad V_{1}^{-\frac{3}{3}} U_{1}^{3}=2 \mu^{\frac{3}{(3}(n+1)} \nu^{-\frac{3}{(3}(m+\beta-1)} f_{3}^{\frac{3}{3}}(\nu) U_{2}$.

This requires either

$$
V_{2}=K \theta(\nu)
$$

or

$$
\theta(\nu)=0 \text {. }
$$

Case $(a)$. If $V_{2}=K \theta(\nu)$, then we find :

which require that

$$
\begin{aligned}
& U_{1}^{3}=\lambda \mu^{-\xi(n+1)}\left[\mu^{-2 n}+2 n^{2} K\right]^{-1}, \\
& U_{1}^{3}=a \mu^{3(n+1)} U_{2},
\end{aligned}
$$

therefore

$$
U_{2}=\frac{\lambda}{a} \mu^{-2(n+1)}\left[\mu^{-2 n}+2 n^{2} K\right]^{-1} ;
$$

And for $V_{1}$ we find:

$$
U_{1}=\frac{\lambda \mu^{n-2}}{\left[2 n^{2} \bar{K} \mu^{2 n}+1\right]^{\frac{3}{2}}} .
$$

These require that:

$$
\begin{aligned}
& V_{1}^{3}=\lambda^{\prime} \nu^{-\frac{3}{3}(m+\beta-1)} f_{3}^{\frac{4}{3}}(\nu) \theta(\nu), \\
& V_{1}^{3}=a^{\prime} \nu^{\frac{4}{(m+\beta-1)}} f_{3}^{-\frac{3}{3}}(\nu) .
\end{aligned}
$$

$$
\theta(\nu)=\frac{a^{\prime}}{\lambda^{\prime}} \frac{\nu^{2(m+\beta-1)}}{f_{3}^{4}(\nu)}
$$

Taking $a^{\prime}=\lambda^{\prime}$, as we may do, and restoring the value of $\theta(\nu)$ we have the following differential equation to determine $f_{3}(\nu)$ : 


$$
\frac{d^{2} f_{3}(\nu)}{d \nu^{2}}-(\beta+m-1) \nu^{-1} \frac{d f_{3}(\nu)}{d \nu}+\beta m \nu^{-2} f_{3}(\nu)=\nu^{2 m+\beta-1} f_{3}^{-3}(\nu)
$$

Before solving this differential equation we consider case $(b)$. If $\theta(\nu)=0$ we find :

giving

$$
\begin{aligned}
& U_{1}^{3}=\lambda \mu^{-\frac{4}{3}(n+1)}, \\
& U_{1}^{3}=a \mu^{\frac{3}{3}(n+1)} U_{2},
\end{aligned}
$$

also

$$
U_{2}=\frac{\lambda}{\mu} \mu^{-2(n+1)} ;
$$

giving

$$
\begin{aligned}
& V_{1}^{\frac{3}{3}}=\lambda^{\prime} \nu^{-\frac{3}{3}(m+\beta-1)} f_{3}^{\frac{3}{3}}(\nu) V_{2}, \\
& V_{1}^{3}=a^{\prime} \nu^{\frac{4}{(m+\beta-1)}} f_{1}^{-\frac{8}{3}}(\nu),
\end{aligned}
$$

And $f_{3}(\nu)$ is given by

$$
V_{2}=\frac{a^{\prime}}{\lambda^{\prime}} \frac{\nu^{2(m+\beta-1)}}{f_{3}^{4}(\nu)} .
$$

$$
\frac{d^{2} f_{3}(\nu)}{d \nu^{2}}-(\beta+m-1) \nu^{-1} \frac{d f_{3}(\nu)}{d \nu}+\frac{\beta m}{\nu^{2}} f_{3}(\nu)=0 .
$$

It remains then to see if $f_{3}(\nu)$ can be determined algebraically.

Since equation (24) is (23) with its right hand number put equal to zero we can consider them together.

We put

$$
\nu=e^{\vartheta}, \quad f_{3}(\nu)=e^{\frac{(m+\beta) \vartheta}{2}} z, \quad \theta=\frac{d z}{d \vartheta} .
$$

After some reduction equations (23) and (24) reduce to the forms :

$$
\begin{aligned}
& \theta \frac{d \theta}{d z}-\frac{(m-\beta)^{2}}{4} z=z^{-3}, \\
& \theta \frac{d \theta}{d z}-\frac{(m-\beta)^{2}}{4} z=0 .
\end{aligned}
$$

We consider the latter and simpler case first.

It gives :

Hence

$$
\theta=\frac{1}{2} \sqrt{(m-\beta)^{2} z^{2}+4 c} .
$$

Therefore

$$
\vartheta=\int \frac{2 d z}{\sqrt{(m-\beta)^{2} z^{2}+4 c}}
$$

$$
\vartheta=\log \left[\frac{2(m-\beta)^{2} z+2(m-\beta) \sqrt{(m-\beta)^{2} z^{2}+4 c_{1}}}{2(m-\beta)^{2} z-2(m-\beta) \sqrt{(m-\beta)^{2} z^{2}+4 c_{1}}}\right]^{\frac{1}{m-\beta}}+\log c_{2} .
$$


Remembering that $\vartheta=\log \nu$ and that $z=f_{3}(\nu) / \nu^{\frac{m+\beta}{2}}$, we find after some reduction :

$$
f_{3}^{2}(\nu)=\frac{4 c_{1} \nu^{m+\beta}}{(m-\beta)^{2}\left\{\left(\nu^{m-\beta}-c_{2}\right)^{2}-1\right\}} ;
$$

and for the equation of the curve:

Hence

$$
(m-\beta)^{2}\left\{\left(\nu^{m-\beta}-c_{3}\right)^{2}-1\right\}\left(A \mu^{n} \nu^{m}+B \mu^{n} \nu^{\beta}\right)^{2}-4 c_{1} \nu^{m+\beta}=0 .
$$

these give :

$$
\begin{aligned}
& V_{1}=K_{1}\left[\nu^{2(m-\beta)-1}-2 c_{2} \nu^{m+\beta-1}+\left(c_{2}^{2}-1\right) \nu^{-1}\right]^{2}, \\
& U_{1}=K_{2} \mu^{-2(n+1)}
\end{aligned}
$$

$$
\begin{aligned}
& E=p \mu^{-2(n+1)}, \\
& G=q\left[\nu^{2(m-\beta)-1}-2 c_{2} \nu^{m-\beta-1}+\left(c_{2}^{2}-1\right) \nu^{-1}\right]^{2},
\end{aligned}
$$

where $p$ and $q$ are constant.

In regard to the other integral:

$$
\vartheta=\int \frac{2 z d z}{2 c z+\sqrt{(m-\beta)^{2} z^{4}-4}},
$$

it appears that in order to keep the exponents rational and the coefficients real it is necessary to take $c=0$.

Then we find finally :

$$
f_{3}^{2}(\nu)=\frac{4 K^{2} \nu^{2 \beta}+\nu^{2 m}}{2 K(m-\beta)}
$$

where $K$ is constant, and the curve is given by the equation :

$$
4 K^{2} \nu^{2 \beta}+\nu^{2 m}=2 K(m-\beta)\left(A \mu^{n} \nu^{m}+B \mu^{n} \nu^{\beta}\right)^{2} .
$$

To revert to the general theory, we may say that if a solution exists, the right hand member of equation 1 of (19) must be of the form

and then

$$
\stackrel{\Theta(\nu)}{\Theta(\mu)},
$$

$$
V_{1}^{3}=K \Theta(\nu), \quad U_{1}^{3}=K \Theta(\mu) .
$$

The functions $\psi_{1}(\mu, \nu)$ and $\psi_{2}(\mu, \nu)$ are given by the equations :

$$
\frac{F_{1}\left(\psi_{1}, \psi_{2}\right)}{\bar{F}_{3}\left(\psi_{1}, \psi_{2}\right)}=\frac{f_{1}(\mu, \nu)}{f_{3}(\mu, \nu)}, \quad \frac{F_{2}\left(\psi_{1}, \psi_{2}\right)}{F_{3}\left(\psi_{1}, \psi_{2}\right)}=\frac{f_{2}(\mu, \nu)}{f_{3}(\mu, \nu)} .
$$

Since we desire a conformal representation we shall take

$$
\psi_{1}=\mu, \quad \psi_{2}=\nu
$$


But it is plain that by such a choice certain conditions are imposed by equations (19) and we proceed to consider those conditions.

$\S 3$.

Calculation of $V_{2}$ and $U_{2}$.

If we represent the right hand members (19) by $R$ and $S$ respectively, then we must have :

1.

$$
R S=1
$$

2.

$$
\frac{\partial^{2} \log R}{\partial \mu \partial \nu}=0 \text {, }
$$

3.

$$
\frac{\partial^{2} \log S}{\partial \mu \partial \nu}=0
$$

From the second of these we find:

$$
\frac{4}{3} \frac{\partial}{\partial \nu} \frac{a_{2}}{a_{5}}-\frac{2}{3} \frac{\partial}{\partial \mu} \frac{a_{3}}{a_{5}}+\frac{\partial^{2} \log \left(F_{3}+V_{2}\right)}{\partial \mu \partial \nu}=0
$$

Considering the last term of this equation we find

since

$$
\begin{aligned}
& \frac{\partial^{2} \log \left(F_{3}+V_{2}\right)}{\partial \mu \partial \nu}=\frac{\partial}{\partial \nu} \frac{\partial F_{3} / \partial \nu}{F_{3}+V_{2}}=\frac{\partial}{\partial \nu}\left\{-4 \frac{a_{4}}{a_{5}} F_{2}^{-2}\left(F_{4}+U_{2}\right)\right\} \\
& =-4 F_{2}^{-2}\left(F_{4}+U_{2}\right) \frac{\partial}{\partial \nu} \frac{a_{4}}{a_{5}}+8 \frac{a_{3}}{a_{5}} \frac{a_{4}}{a_{5}} F_{2}^{-2}\left(F_{4}+U_{2}\right)-4 \frac{a_{1}}{a_{5}} \frac{a_{4}}{a_{5}}
\end{aligned}
$$

$$
\frac{\partial F_{3}}{\partial \mu}=-\frac{a_{4}}{a_{5}} F_{1}^{-2} \quad \text { and } \quad 4 F_{1}^{2} F_{2}^{-2}\left(F_{3}+V_{2}\right)\left(F_{4}+U_{2}\right)=1
$$

Whence from (28) we find :

$$
2 F_{2}^{-2}\left(F_{4}+U_{2}\right)=\frac{\frac{2}{3} \frac{\partial}{\partial \mu} \frac{a_{3}}{a_{5}}-\frac{4}{3} \frac{\partial}{\partial \nu} \frac{a_{2}}{a_{5}}+4 \frac{a_{1}}{a_{5}} \frac{a_{4}}{a_{5}}}{4 \frac{a_{3}}{a_{5}} \frac{a_{4}}{a_{5}}-2 \frac{\partial}{\partial \nu} \frac{a_{4}}{a_{5}}}
$$

and from 3 of (27) we find in like manner:

$$
2 F_{1}^{2}\left(F_{3}+V_{2}\right)=\frac{\frac{4}{3} \frac{\partial}{\partial \mu} \frac{a_{3}}{a_{5}}-\frac{2}{3} \frac{\partial}{\partial \nu} \frac{a_{2}}{a_{5}}+4 \frac{a_{1}}{a_{5}} \frac{a_{4}}{a_{5}}}{4 \frac{a_{1}}{a_{5}} \frac{a_{2}}{a_{5}}+2 \frac{\partial}{\partial \mu} \frac{a_{1}}{a_{5}}}
$$

If we represent the right hand members of (29) and (30) by $\Delta_{1}$ and $\Delta_{2}$ respectively, then equations (19) become: 


$$
\begin{aligned}
& \frac{V_{1}^{3}}{U_{1}^{\frac{3}{4}}}=F_{1}^{-\frac{3}{3}} F_{2}^{-\frac{3}{3}} \Delta_{1}, \\
& \frac{U_{1}^{\frac{3}{3}}}{V_{1}^{\frac{3}{3}}}=F_{1}^{3} F_{2}^{3} \Delta_{2} .
\end{aligned}
$$

$\S 4$.

Partial differential equations satisfied by $f_{i}(\mu, \nu)$.

As a first condition we have

$$
\Delta_{1} \Delta_{2}=1
$$

Differentiating (29) with respect to $\nu$, and using (19) we have

or

$$
2 \frac{a_{1}}{a_{5}} F_{2}^{2}=F_{2}^{2} \frac{\partial \Delta_{1}}{\partial \nu}+2 F_{2}^{2} \frac{a_{3}}{a_{5}} \Delta_{1}
$$

$$
\frac{\partial \Delta_{1}}{\partial \nu}+2 \frac{a_{3}}{a_{5}} \Delta_{1}=2 \frac{a_{1}}{a_{5}}
$$

and in like manner from (30) we find

$$
\frac{\partial \Delta_{2}}{\partial \mu}-2 \frac{a_{2}}{a_{5}} \Delta_{2}=-2 \frac{a_{4}}{a_{5}}
$$

Hence we have the system :

$$
\begin{aligned}
& \Delta_{1} \Delta_{2}=1, \\
& \frac{\partial \Delta_{1}}{\partial \nu}+2 \frac{a_{3}}{a_{5}} \Delta_{1}=2 \frac{a_{1}}{a_{5}}, \\
& \frac{\partial \Delta_{2}}{\partial \mu}-2 \frac{a_{2}}{a_{5}} \Delta_{2}=-2 \frac{a_{4}}{a_{5}} .
\end{aligned}
$$

These are the necessary, and, as is easily seen, sufficient conditions to impose upon $f_{i}(\mu, \nu)$ in order that a solution may exist for the rectangular coördinate system $(\mu, \nu)$.

\section{$\S 5$.}

We consider next a somewhat general solution of our problem. We have: written

$$
\int \frac{a_{2}}{a_{5}} d \mu=\log F_{1}, \quad \int \frac{a_{3}}{a_{5}} d \nu=\log F_{2} .
$$

We consider one case where these integrals actually yield logarithms, i. e., $\partial a_{5} / \partial \mu$ $=\lambda a_{2}$ and $\partial a_{5} / \partial \nu=a a_{3}$. To simplify the expressions for $a_{i}$ we divide equation 
(2) through by $f_{2}(\mu, \nu)$ and then consider $f_{3}^{\prime}=1, f_{2}^{\prime}=f_{2} / f_{3}, f_{3}^{\prime}=f_{1} / f_{3}$. This amounts to putting $f_{3}=1$ in our formulas. We find:

$$
\begin{gathered}
a_{1}=\left|\begin{array}{ll}
\frac{\partial f_{2}}{\partial \mu} & \frac{\partial^{2} f_{2}}{\partial \mu^{2}} \\
\frac{\partial f_{3}}{\partial \mu} & \frac{\partial^{2} f_{3}}{\partial \mu^{2}}
\end{array}\right|, \quad a_{4}=\left|\begin{array}{ll}
\frac{\partial f_{2}}{\partial \nu} & \frac{\partial^{2} f_{2}}{\partial \nu^{2}} \\
\frac{\partial f_{3}}{\partial \nu} & \frac{\partial^{2} f_{3}}{\partial \nu^{2}}
\end{array}\right|, \quad a_{5}=\left|\begin{array}{ll}
\frac{\partial f_{2}}{\partial \mu} & \frac{\partial f_{2}}{\partial \nu} \\
\frac{\partial f_{3}}{\partial \mu} & \frac{\partial f_{3}}{\partial \nu}
\end{array}\right|, \\
a_{2}=2\left|\begin{array}{ll}
\frac{\partial f_{2}}{\partial \mu} & \frac{\partial^{2} f_{2}}{\partial \mu \partial \nu} \\
\frac{\partial f_{3}}{\partial \mu} & \frac{\partial^{2} f_{3}}{\partial \mu \partial \nu}
\end{array}\right|+\left|\begin{array}{ll}
\frac{\partial f_{2}}{\partial \nu} & \frac{\partial^{2} f_{2}}{\partial \mu^{2}} \\
\frac{\partial f_{3}}{\partial \nu} & \frac{\partial^{2} f_{3}}{\partial \mu^{2}}
\end{array}\right|, \quad a_{3}=2\left|\begin{array}{ll}
\frac{\partial f_{2}}{\partial \nu} & \frac{\partial^{2} f_{2}}{\partial \mu \partial \nu} \\
\frac{\partial f_{3}}{\partial \nu} & \frac{\partial^{2} f_{3}}{\partial \mu \partial \nu}
\end{array}\right|+\left|\begin{array}{ll}
\frac{\partial f_{2}}{\partial \mu} & \frac{\partial^{2} f_{2}}{\partial \nu^{2}} \\
\frac{\partial f_{3}}{\partial \mu} & \frac{\partial^{2} f_{3}}{\partial \nu^{2}}
\end{array}\right| .
\end{gathered}
$$

The case to be considered is found by taking $\partial^{2} f_{2} / \partial \mu \partial \nu=0$ and $\partial^{2} f_{3} / \partial \mu \partial \nu=0$, that is, $f_{2}=\phi_{1}(\mu)+\phi_{2}(\nu)$ and $f_{3}=\psi_{1}(\mu)+\psi_{2}(\nu)$. Then we shall have:

Hence

$$
\begin{aligned}
& a_{5}=\phi_{1}^{\prime}(\mu) \psi_{2}^{\prime}(\nu)-\psi_{1}^{\prime}(\mu) \phi_{2}^{\prime}(\nu), \\
& a_{1}=\phi_{1}^{\prime}(\mu) \psi_{1}^{\prime \prime}(\mu)-\psi_{1}^{\prime}(\mu) \phi_{1}^{\prime \prime}(\mu), \\
& a_{4}=\phi_{2}^{\prime}(\nu) \psi_{2}^{\prime \prime}(\nu)-\psi_{2}^{\prime}(\nu) \phi_{2}^{\prime \prime}(\nu), \\
& a_{2}=\phi_{2}^{\prime}(\nu) \psi_{1}^{\prime \prime}(\mu)-\psi_{2}^{\prime}(\nu) \phi_{1}^{\prime \prime}(\mu), \\
& a_{3}=\phi_{1}^{\prime}(\mu) \psi_{2}^{\prime \prime}(\nu)-\psi_{1}^{\prime}(\mu) \phi_{2}^{\prime \prime}(\nu) .
\end{aligned}
$$

Therefore

$$
\int \frac{a_{2}}{a_{5}} d \mu=-\log \left\{\phi_{1}^{\prime}(\mu) \psi_{2}^{\prime}(\nu)-\psi_{1}^{\prime}(\mu) \phi_{2}^{\prime}(\nu)\right\},
$$

Hence

$$
F_{1}=1 \div\left|\begin{array}{ll}
\phi_{1}^{\prime}(\mu) & \phi_{2}^{\prime}(\nu) \\
\psi_{1}^{\prime}(\mu) & \psi_{2}^{\prime}(\nu)
\end{array}\right|=\frac{1}{a_{5}}, \quad F_{2}=\left|\begin{array}{ll}
\phi_{1}^{\prime}(\mu) & \phi_{2}^{\prime}(\nu) \\
\psi_{1}^{\prime}(\mu) & \psi_{2}^{\prime}(\nu)
\end{array}\right| \because a_{5} .
$$

$$
\begin{aligned}
& F_{3}^{\prime}=\int-\frac{a_{4}}{a_{5}} F_{1}^{-2} d \mu=-a_{4} \int a_{5} d \mu=-\left|\begin{array}{ll}
\phi_{1}(\mu) & \phi_{2}^{\prime}(\nu) \\
\psi_{1}(\mu) & \psi_{2}^{\prime}(\nu)
\end{array}\right| \cdot\left|\begin{array}{ll}
\phi_{2}^{\prime}(\nu) & \phi_{2}^{\prime \prime}(\nu) \\
\psi_{2}^{\prime}(\nu) & \psi_{2}^{\prime \prime}(\nu)
\end{array}\right|, \\
& F_{4}=\int \frac{a_{1}}{a_{5}} F_{2}^{2} d \nu=a_{1} \int a_{5} d \nu=\left|\begin{array}{ll}
\phi_{1}^{\prime}(\mu) & \phi_{2}(\nu) \\
\psi_{1}^{\prime}(\mu) & \psi_{2}(\nu)
\end{array}\right| \cdot\left|\begin{array}{ll}
\phi_{1}^{\prime}(\mu) & \phi_{1}^{\prime \prime}(\mu) \\
\psi_{1}^{\prime}(\mu) & \psi_{1}^{\prime \prime}(\mu)
\end{array}\right| .
\end{aligned}
$$

In order to separate the variables it is necessary that either
(a) $\phi_{1}(\mu)=\psi_{1}(\mu)$ or
(b) $\phi_{2}(\nu)=\psi_{2}(\nu)$ 
and since the equations and expressions are symmetrical in $\mu$ and $\nu$ it will only be necessary to consider case (a) and afterwards interchange $\mu$ and $\nu$ in the result.

First of all we have:

$$
\begin{gathered}
F_{4}=0, F_{1}=\frac{1}{\phi^{\prime}(\mu)} \frac{1}{\psi_{2}^{\prime}(\nu)-\phi_{2}^{\prime}(\nu)}, F_{2}=\phi^{\prime}(\mu)\left\{\psi_{2}^{\prime}(\nu)-\phi_{2}^{\prime}(\nu)\right\} \\
F_{3}=-\phi(\mu)\left\{\psi_{2}^{\prime}(\nu)-\phi_{2}^{\prime}(\nu)\right\}\left|\begin{array}{ll}
\phi_{2}^{\prime}(\nu) & \phi_{2}^{\prime \prime}(\nu) \\
\psi_{2}^{\prime}(\nu) & \psi_{2}^{\prime \prime}(\nu)
\end{array}\right| .
\end{gathered}
$$

Then from (19) we have:

$$
\frac{\frac{1}{2} \phi^{\prime 2}(\mu)\left\{\boldsymbol{\psi}_{2}^{\prime}(\nu)-\phi_{2}^{\prime}(\nu)\right\}}{\frac{V_{2}}{\left\{\boldsymbol{\psi}_{2}^{\prime}(\nu)-\phi_{2}^{\prime}(\nu)\right\}}-\phi(\mu)\left|\begin{array}{ll}
\phi_{2}^{\prime}(\nu) & \phi_{2}^{\prime \prime}(\nu) \\
\psi_{2}^{\prime}(\nu) & \psi_{2}^{\prime \prime}(\nu)
\end{array}\right|}=\frac{2 U_{2}}{\phi^{\prime 2}(\mu)\left\{\boldsymbol{\psi}_{2}^{\prime}(\nu)-\phi_{2}^{\prime}(\nu)\right\}} .
$$

As $V_{1}$ must vanish and $U_{2}=\phi_{1}^{\prime 4}(\mu) / 4 \phi(\mu)$, we find

Also

$$
U_{1}=\frac{1}{\sqrt{8}} \frac{\phi^{\prime 3}(\mu)}{\phi^{3}(\mu)}
$$

$$
\left|\begin{array}{ll}
\phi_{2}^{\prime \prime}(\nu) & \phi_{2}^{\prime}(\nu) \\
\psi_{2}^{\prime \prime}(\nu) & \psi_{2}^{\prime}(\nu)
\end{array}\right|=\left\{\psi_{2}^{\prime}(\nu)-\phi_{2}^{\prime}(\nu)\right\}^{3}
$$

or, if we write $x$ for $\psi_{2}^{\prime}(\nu)$ and $y$ for $\phi_{2}^{\prime}(\nu)$,

$$
x \frac{d y}{d \nu}-y \frac{d x}{d \nu}=(x-y)^{3} .
$$

Put $x-y=q$ and our differential equation is :

$$
q \frac{d x}{d \nu}-x \frac{d q}{d \nu}=q^{3}
$$

or

Therefore

$$
\frac{d}{d \nu} \frac{x}{q}=q
$$

hence

$$
\frac{x}{q}=\int q d \nu=\psi_{2}(\nu)-\phi_{2}(\nu)+c ;
$$

$$
\frac{\psi_{2}^{\prime}(\nu)}{\psi_{2}^{\prime}(\nu)-\phi_{2}^{\prime}(\nu)}=\psi_{2}(\nu)-\phi_{2}(\nu)+c_{1}
$$

and integrating this we have:

$$
\psi_{2}(\nu)=\frac{1}{2}\left[\psi_{2}(\nu)-\phi_{2}(\nu)\right]^{2}+c_{1}\left[\psi_{2}(\nu)+\phi_{2}(\nu)\right]+c_{2} .
$$


Remembering that $\phi_{2}(\nu)=f_{1}(\nu) / f_{3}(\nu)$ and $\phi(\mu)=f_{1}(\mu) / f_{3}(\mu)$ we have, after some reduction :

$$
\begin{aligned}
& B^{2}\left[K_{2} f_{1}(\nu)+K_{3} f_{3}(\nu)\right] f_{3}(\nu) f_{3}^{2}(\mu) \\
& \quad=\left\{\left(K_{1} B+1\right) f_{3}(\nu) f_{3}(\mu)+(A+B)\left[f_{1}(\mu) f_{3}(\nu)+f_{3}(\mu) f_{1}(\nu)\right]\right\}^{2},
\end{aligned}
$$

for the equation of our curve. Here the $f_{i}$ of $\mu$ and of $\nu$ are any algebraic functions whatever. As mentioned above we find a solution for case (b) by in. terchanging $\mu$ and $\nu$ in (43).

$\S 6$.

Conditions for the equality of $E$ and $G$.

The work thus far is for $F=0$, i. e., $\mu$ and $\nu$ are rectangular surface coördinates. It remains to consider the case $E=G$.

From our system of partial differential equations (10) we find that $a_{1}$ must be equal to $a_{3}$ and $a_{2}$ to $a_{4}$. It remains to consider the system of equations (32) which were the necessary and sufficient conditions to impose on the $f_{i}(\mu, \nu)$. First we consider the values of $F_{3}$ and $F_{4}$. We recall that

$$
\begin{gathered}
F_{3}=\int-\frac{a_{4}}{a_{5}} F_{1}^{-2} d \mu, \quad F_{4}=\int \frac{a_{1}}{a_{5}} F_{2}^{-2} d \nu, \\
\log F_{1}=\int \frac{a_{2}}{a_{5}} d \nu, \quad \log F_{2}=\int \frac{a_{3}}{a_{5}} d \nu .
\end{gathered}
$$

Hence also

We have then

$$
\frac{\partial F_{1}}{\partial \mu}=\frac{a_{2}}{a_{5}} F_{1}, \quad \frac{\partial F_{2}}{\partial \nu}=\frac{a_{3}}{a_{5}} F_{2}
$$

$$
F_{3}=\int-\frac{a_{4}}{a_{5}} F_{1}^{-2} d \mu=\int-\frac{a_{2}}{a_{5}} F_{1}^{-2} d \mu=\int-F_{1}^{-3} \frac{\partial F_{1}}{\partial \mu} d \nu=\frac{1}{2} F_{1}^{-2}
$$

Also

$$
F_{4}=\int \frac{a_{3}}{a_{5}} F_{2}^{2} d \nu=\int F_{2} \frac{\partial F_{2}}{\partial \nu} d \nu=\frac{1}{2} F_{2}^{2}
$$

Hence the right hand members of $(29)$ and (30) become respectively,

$$
2 F_{2}^{-2}\left(\frac{1}{2} F_{2}^{2}+U_{2}\right)=1+2 F_{2}^{-2} U_{2}=\Delta_{1}=\frac{\frac{2}{3} \frac{\partial}{\partial \mu} \frac{a_{3}}{a_{5}}-\frac{4}{3} \frac{\partial}{\partial \nu} \frac{a_{2}}{a_{5}}+4 \frac{a_{2}}{a_{5}} \frac{a_{3}}{a_{5}}}{4 \frac{a_{2}}{a_{5}} \frac{a_{3}}{a_{5}}-2 \frac{\partial}{\partial \nu} \frac{a_{2}}{a_{5}}},
$$




$$
2 F_{1}^{2}\left(\frac{1}{2} F_{1}^{-2}+V_{2}\right)=1+2 F_{1}^{2} V_{2}=\Delta_{2}=\frac{\frac{4}{3} \frac{\partial}{\partial \mu} \frac{a_{3}}{a_{5}}-\frac{2}{3} \frac{\partial}{\partial \nu} \frac{a_{2}}{a_{5}}+4 \frac{a_{2}}{a_{5}} \frac{a_{3}}{a_{5}}}{4 \frac{a_{2}}{a_{5}} \frac{a_{3}}{a_{5}}+2 \frac{\partial}{\partial \mu} \frac{a_{3}}{a_{5}}} .
$$

We consider the first of equations (3), i. e., $\Delta_{1} \Delta_{2}=1$.

We write

and also

$$
\Delta_{1}=\frac{\frac{2}{3} a-\frac{4}{3} b+c}{c-2 b}=1+\frac{\frac{2}{3}(a+b)}{c-2 b}
$$

Therefore

$$
\Delta_{2}=\frac{\frac{4}{3} a-\frac{2}{3} b+c}{c+2 a}=1-\frac{\frac{2}{3}(a+b)}{c+2 a} .
$$

$$
\begin{aligned}
\Delta_{1} \Delta_{2} & =1+\frac{\frac{2}{3}(a+b)}{c-2 b}-\frac{\frac{2}{3}(a+b)}{c+2 a}-\frac{\frac{4}{9}(a+b)^{2}}{(c+2 a)(c-2 b)} \\
& =1+\frac{\frac{2}{3}(a+b)}{(c+2 a)(c-2 b)}\left[c+2 a-c+2 b-\frac{2}{3}(a+b)\right] \\
& =1+\frac{\frac{8}{9}(a+b)}{(c+2 a)(c-2 b)} .
\end{aligned}
$$

Hence we must have $a+b=0$ and therefore

$$
\frac{\partial}{\partial \mu} \frac{a_{3}}{a_{5}}+\frac{\partial}{\partial \nu} \frac{a_{2}}{a_{5}}=0
$$

Hence $\Delta_{1}=1$ and $\Delta_{2}=1$. Then the other two equations of (32) are satisfied and we may conclude that

$$
\begin{aligned}
& a_{1}=a_{3}, \\
& a_{2}=a_{4}, \\
& \frac{\partial}{\partial \mu} \frac{a_{3}}{a_{5}}+\frac{\partial}{\partial \nu} \frac{a_{2}}{a_{5}}=0,
\end{aligned}
$$

are the necessary and sufficient conditions that $E=G$.

The next question will be the study of these surfaces, their existence and properties. In particular I have already finished part of the work for the curve $y^{2}=a x^{3}+b x^{2}+c x+d$, i. e., for surfaces whose geodetic lines go into cubics upon the plane by a conformal transformation. The straight line and circle lead to the surfaces of constant curvature with their interesting properties. Here is a much wider and more interesting field.

GöTrINGEN, November 3, 1900. 